\title{
Representation of Screen Marbling Techniques with Reactive and Vat Dyes Combination on Textiles and Fashion Design Products for the Ghanaian Market
}

\author{
Bernard Edem Dzramedo $^{1 *} \quad$ Robert $_{\text {Ahiabor }}^{2}$ \\ 1.Department of Family and Consumer Sciences, Faculty of Agriculture, University for Development Studies \\ (UDS), P. O. Box TL 1882, Tamale - N/R, Nyankpala Campus, Ghana \\ 2.Department of Fashion Design and Modelling, School of Applied Art, Design and General Studies, Wa \\ Polytechnic, P. O. Box 553, U/W, Ghana
}

\begin{abstract}
Textile products are not only patronised for their functionality and communicative purposes, but equally for their aesthetics. Marbling has been a cherished art for centuries with attractive and intricate designs used for decorative purposes. The study explores the possibility of transferring marbling designs using reactive and vat dyes combination through screen printing method to produce fabrics that can be fashioned into garments and accessories. The study employed both descriptive and experimental methods to assess market trends of existing fabric designs in selected markets in the country and then produce marbling effects using vat and reactive dyes to popularise the art for everyday usage in fabric, garments and accessories. Mercerized cotton was used as based material for the printing processes which were fashioned into garments and accessories. The market survey on existing fabric designs revealed non-availability of designs reflecting the silk screen marbling effects using vat dyes as print paste on reactive dyed backgrounds in the Ghanaian market, hence paving the way for the experiment to that effect. The results from the experiment established that the fluidity of the marbling designs with its colour integration in aesthetic manner makes it favourable to compete in the fabric market. This, the researchers believed forms the basis for more further study in exploring related arts as means of expanding creativity and creating jobs for the youth. The recommendation was mainly on further study to improve on colour matching techniques for enhanced and varied outcomes.
\end{abstract}

Keywords: Aesthetics, Marbling techniques, African prints, Vat and Reactive Dyes

DOI: $10.7176 / \mathrm{ADS} / 80-05$

Publication date: January $31^{\text {st }} 2020$

\section{Introduction}

Marbling dated years back into history and covers the art of bookbinding, wall paper decorating piece lamp shades and gift wrappers among others. However, the prospects in the art goes beyond these traditional uses into various items of both functional and aesthetic purposes (Pazar, 2016). Today marbling is equally associated with fabric decoration which is effected by floating fabric paints or pigments on think cellulosic (sizing) solution like oil on water where the paints are swirled into patterns and the design captured by laying the treated fabric on top of the paint to transfer the swirls to the fabric (Dharma trading co., 2019; Batik-Octoro, 2019). In the context of this study, the designs are not transferred directly to the fabric but rather those designs are captured on paper and transferred into printable patterns on screens for repeated printing using reactive and vat dyes use medium.

The art of marbling was a cherished tradition in Asia and Europe for centuries and was used for decorative purposes as well as background texture for official documents and signatures, to prevent erasure and forgery. The art of marbling was an essential part of bookbinding and a secret held tradition within family practitioners till the mid-20 $0^{\text {th }}$ century (Galen, 2015). Although this art is not popular in the field of fabric production generally and particularly in Ghana, evidence of its use exist in local fabric works in two forms using paper marbling technique and tie-dye marbling techniques (Howard, 2013). Marbling techniques in screen printing process will encourage the reproduction of designs when the need be which is a difficult process to get if not impossible with the other forms of marbling techniques. This screen printing technique is to be explored using vat as print paste on reactive dyed backgrounds. The purpose is to discharge the reactive dyes and redeposit the vat dyes. Its intention is to provide avenue for new exploit in this direction for the local textile industry. Humphries (2004) defines printing as the application of a design, whether coloured or not, using dyes, pigment, usually in a thick paste, bound to the surface of a fabric or other substances. Tortora and Phyllis (2007) contend that printing is the application of colourants in definite, repeated patterns to fabric, yarn or sliver by any one of a number of methods other than dyeing.

In view of this developments regarding marbling art, the researchers sought to employ the screen printing technique of achieving similar marbled effects on textile substrates which can be sewn into fashion design products. This exploration of marbled effects would go a long way to add to existing fabric design techniques by small and medium scale printing industries and provide wider variations of textile designs that will meet the 
demand of the textile market.

Objective: The general objective is to identify trends of African print designs in the Ghanaian market with similarity to marbling as bases for the designing and production of fabrics using discharge marbling techniques from vat and reactive dyes for apparels and its accessories.

\section{Specific objectives:}

- To assess types of African prints with marbling characteristics in the Ghanaian market and their rate of patronage.

- $\quad$ To prepare a print paste using vat dyes with local starches acting as thickening agents for screen printing process.

- To achieve a marbled effect with vat dyes as paste on reactive dyed cotton fabric using discharge technique through screen printing method.

- To assess the acceptability of vat dyes paste - screen printed product in the Ghanaian market by fashioning the fabrics into garments and accessories.

Purpose: The purpose of the study is to explore new grounds by using dyes as paste to enhance screen printing processes for easier, cheaper but durable fabric production to improve on their marketing chances.

Significance: The study enhanced on the production of marbling techniques in fabric designing using screen printing method with vat and reactive dyes as its main ingredients. This is to encourage and open creative ways for practitioners in the field of printing and dyeing by creating more options for students, researchers in the field of textiles and fashion design.

\section{Literature Review}

The review intends to understand the level of work done in this area with references to marbling art in general and its relevance in the field of screen printing using vat dye paste on reactive dyed background.

\section{Fabric Surface Design and Art}

Design and art are mostly fused in their use concurrently in most instances in art related subjects. Design refers to a plan within a work of art. It is seen as an organization, arrangement or composition of a work. This implies that design can be considered as a process or as the result of a process (Asante and Nyarko-Hibson, 2008). The creative process involves conveying or translating ideas that have been conceived in the mind's eye into tangible form through the use of suitable tool/tools and medium/media. New designs are the outcome of the mind's emotional desires to develop new plans and methods and to formulate devices and ideas for expression. Design concepts emanates from various sources including nature, artificial objects, traditional symbols, proverbs, daily activities and from imaginative composition especially in Ghanaian setting (Asare, 2010; Howard, 2013a; Adu Akwaboa, 1989). Harod and Pomeroy as cited in Manu (2010) ascertain that the word design has many depths of meaning and that only philosophical method will strip off all the meanings to provide coherent and comprehensive views.

\section{Fabric Marbling Art}

Fabric marbling is the art of decorating fabric by application of two or more colours on a fabric in a wet condition so that the colours mixed up to produce varied range of colour tones with irregular and accidental designs (Howard, 2013b). However, a much conscious effort may be employed to produce somewhat semi realistic or a well-defined design. Fabric marbling can be done in two ways. One way is by employing paper marbling technique and the other is by the use of tie-dye marbling technique. Surfaces used for marbling works are essential to the success and look of the finished fabric. According to Bette (1997), a variety of washable fabrics can be used for marbling. Natural fibres work best, although blends may also be used. Chambers (1995) agrees that soft, swirling, elegant marbled effects on silk, satin, cotton and synthetics can now be as easily achieved on fabric as on paper.

\section{Dyeing and Screen Printing Techniques}

The process of producing colours and designs on a fabric is called dyeing and printing, respectively. Wolfe (1989) identified dyeing as a method of giving colour to a fibre, yarn, fabric or garment which carried out with colouring agent called dyes whereas printing involves adding colour, pattern or design to the surface of fabrics. It is very important for the dyed and printed fabrics to be 'colourfast' that is the colour should not come out or fade easily. If the colour runs on washing, rubbing or ironing, the fabric looks shabby and old and its design becomes dull or smudged. The colour may also spoil other fabrics during washing (Kadolph, 1993).

Dyeing and printing improve appearance of fabric and add diversity to our dresses through colours and designs. Fabrics are usually distinguished from one another by colour, print and texture. Wilson (2001) is of the view that CAD systems improve on print designers' ideas readily as it helps in easy manipulations. Many systems also have colour separation facilities to aid in the preparation of screens. Some systems even allow sample fabrics to be produced, and this is a very valuable tool, making it much easier for print designers to transfer ideas onto fabric. 


\section{Clothing and Accessories}

According to Wolf (1989) clothing includes all the garment, accessories and ornaments worn by people throughout the world though they might come in various forms and presentation. Its purpose is to cover the body, communicate factors and project individual personality. They form first impressions which are considered as lasting impression in most instances. Clothes are seen as decorative adornment and communicate cultural and social phenomenon that are expressive in the context of the Africa and as well communicate customs, values and traditions that are significant to any traditional setting. Clothes are used by many societies as non-verbal forms of communicating ranks, wealth, status and identity, representing free form of speech (Weston, 2006; Dzramedo, et al, 2013). According to Shaeffer (2001) apparels construction in most cases must consider three major aspects of its processes; firstly, it must have good design in terms of fabric and style and secondly, it must have a unique and good construction and finishing processes and finally, it must meet market demands.

\section{Marketing Prospects and Strategies}

Quite a number of researchers have identified marketing as key to the success of any new innovation. Akwaboa ( 1989) ascertain that, no matter how important and innovative a product is, without the right type of marketing strategy to appeal to the right customers, an innovative idea will not achieve the desired results. In the realm of marketing, Singh (2006) emphasized on the important role colour plays in packaging, branding and general promotion of products. The right choice of colour for a product must be in conformity with the target audience in question (adults, youth, and children) and must be specific if necessary. This should go with selecting the right mode of advertisement for the product. Fatma (2015) stated that, it is a way of passing on information about a product to generate interest and growing desire in the minds of consumers towards the buying of a product. This he believed is a way marketers are employing to catch hold of consumer's attention toward new products.

\section{Materials and Methods}

\section{Study Area and Preliminary Investigation}

The study areas were mainly Accra, Kumasi and Tamale, considered as metropolitan cities with good geographical representation across the country. The main fabric markets in these cities were visited to assess the types of African prints available with similarities to marbling fabrics based on fluidity of designs and printing methods used. Observation was a major instrument used to assess the types of African prints available with few unstructured interviews carried out with store attendants' and customers who patronized fabrics within the scope of the study. This is to determine traffic flow for such goods and reasons for their purchase. Most of the responses from the consumers were based on the aesthetic appeal of the fabrics and their functional significance as Ghanaians want to be associated with the non-verbal communicative aspects of fabrics. The simple survey also reveals interest is geared towards bright colours and monochrome effects of fabric colours while all the fabric prints are done in the industry using printing inks at the industrial level. This therefore paves way for the researchers to carry out their study.

\section{Research Design and Sampling}

The research began with exploratory study, since the combination of reactive and vat dyes as printing medium are not a known field of art in Ghana. The researchers after gaining more grounds in their exploit used descriptive and experimental research designs for the study. The descriptive method was used to record, describe, analyse and interpret secondary data from books relevant to the study (Best, 1981) and also interpret the procedures involved in the production processes of the study. The experimental research method on the other hand was employed to find the possibility of producing attractive user friendly fabrics using vat dyes as print paste in the presence of locally prepared thickening agents to discharge on reactive dyed fabric using hand screen printing method.

Different kinds of tools and materials were employed in the designing and production of finished garments and accessories made from vat based printed fabrics. Tools according to Brett (1990) are devices used for making items in a workshop. The experiments were carried out in both the researchers' personal studios and their departmental studios as well. By the design of this study, the tools and materials used includes Mercerized cotton, Common salt, Soda ash, Reactive dyes, Rubber basins and spoons, Vat dyes, Squeegee, Silk Screens, Cassava starch, Flour starch, Water, Corn flour, Digital camera, Sewing threads, Sewing machine, Containers, Printer, Computer with Photoshop application, Hand gloves, Plastic tea spoon and Stapler.

\section{Procedures for Fabric Production and Construction}

Designing Process

This experiment started by generating designs based on marbling techniques. Various marbled paper designs were produced out of which some were snapped with a digital camera and edited using Adobe Photoshop software (Figure 1). This software was used to facilitate editing of the marbled art works by checking repeat units, enhancing colour matching and separate all colours for printing of di-positives necessary for development of the screens. The individual colours were converted to black and printed on tracing paper. Silk screen was prepared for the design, stretched to a drum-beat effect with the surface washed and dried to remove any form of 
grease that may hinder the smooth process of copying and development of the work. The screen was then coated with photo emulsion and dried in the dark room before copying and developing were done using light box to transfer the designs from the paper surface onto the screens. The screens were then washed and dried. Finally, pin holes were checked and blocked and the screens masked at the edges with masking tapes ready for printing.
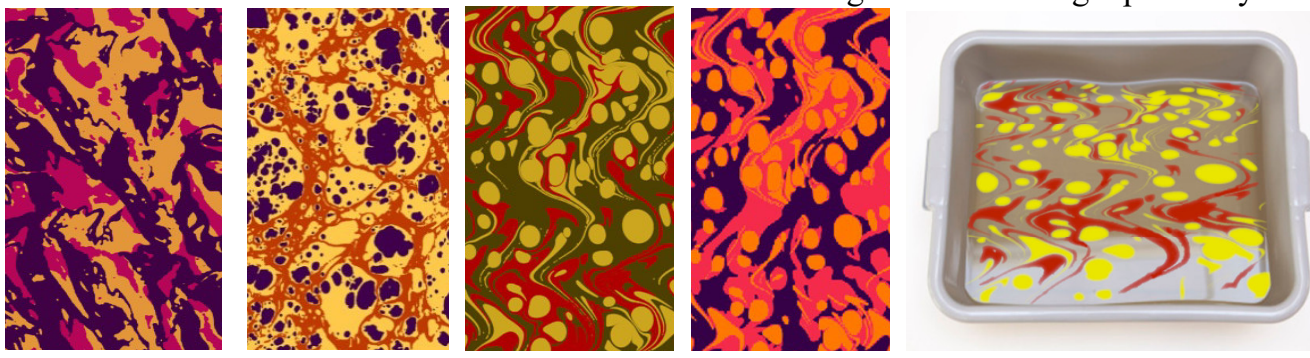

\section{Dyeing with Reactive Dyes}

Dyeing was the next process carried out in the researchers' quest to achieve the intended results. Since the aim of this experiment was to achieve marbled effects using vat dyes on reactive background employing the screen printing method, it became necessary to dye the cotton fabric with reactive dyes of different colours (Figure 2 a \& b). The ratio for the composition of the dye bath was 2:1:6 for $1 / 2$ yard of cotton fabric. This implies 2 tea spoonsful of reactive dyes, 1 tea spoonful of soda ash and 6 tea spoonsful of the common salt prepared into a solution using half cup of water. The fabric is then soaked in water to open the pores of the fibres to increase their receptivity of the dye molecules. The fabric was then immersed in the dye bath prepare from 2 litres of water for 30 minutes (more or less) depending on the intended shade of colour required. The longer the fabric stays in the bath, the deeper the shade obtained. Since vat dyes were printed on the reactive dye background, it was prudent to obtain deeper shades of colours so that the reaction between the reactive dye and the vat dye would be improved. The fabric was later removed from the bath and dried under a shade and then later rinsed from excess dyes that stayed on the surface.
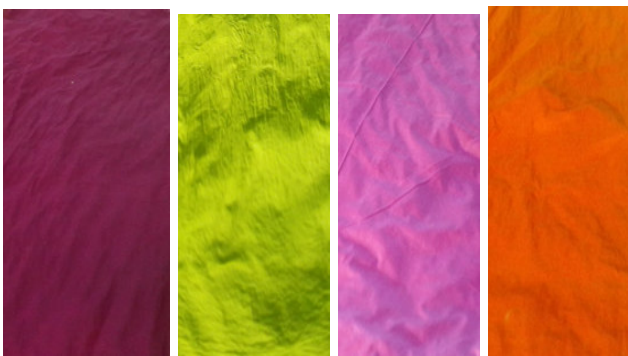

Fig. 2a: Samples of reactive dyed backgrounds used for the experiment.

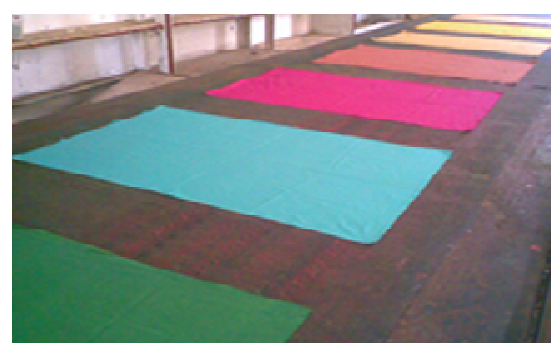

Fig. 2b: Samples stretched on table ready for printing.

Preparing the Vat Dye Paste

The preparation of the vat dye paste forms an important aspect of the process prior to printing of the fabric samples. In preparing the vat dye paste, a concentrated vat dye leuco was prepared using the desired colours. This was done in a ratio of 2:4:3 representing 2 tea spoonsful of vat dye to 4 tea spoonsful of sodium hydrosulphite and 3 tea spoonsful of sodium hydroxide in the presence of small quantity of water so as to paste the dye. This should be enough to print half yard of fabric. The experiment was carried out with vat leuco pasted with samples of thickening agents from cassava starch, corn starch and wheat flour starch (Figure $3 a$ \& b). However, it was clear that the results from wheat flour starch paste produces the best outcomes after printing various samples; probably resulting from the smoothness of the paste it produced. 8 tea spoonful of the wheat flour starch was then added to the vat leuco to paste it ready for printing. Other series of exploration revealed that addition of 2 spoonsful of bleaching agent enhances the end print produced as it aids in discharging of the reactive background for the deposition of the vat dye paste.
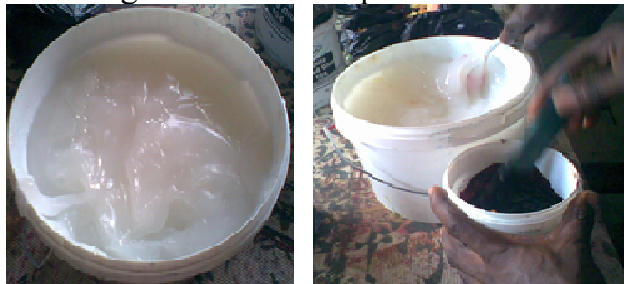

Fig. 3a: Adding cassava Starch as thickening agent to the vat leuco
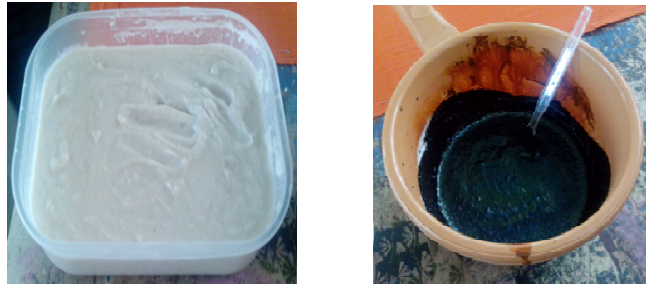

Fig 3b: Wheat flour starch and vat leuco 


\section{Printing Vat Dyes on Reactive Dyed Fabric}

Printing of the fabric was the next process after dyeing and preparation of the vat dye paste. The fabrics were stretched on the printing table and stapled onto the surface. The screens were later positioned on the fabric and printed with the prepared paste using squeegee. In a process where one colour is printed first followed by the other. In the course of printing the repeating units must be positioned well with care for good repeat fitting. The paste is then poured into the reservoir portion of the screen and then squeegeed to transfer the print design onto the fabric (Figure $4 a \& b$ ). After the printing process, the fabrics were dried under shade for about 30 minutes for full oxidation to take place before washing them to remove excess dyes and exposed them for their true colours to emerge (as colours printed initially will not reveal its colours as in the case with vat dyes). The fabric was later dried and ironed (Figure 5).

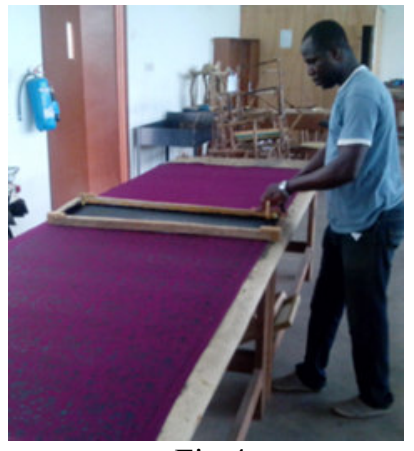

Fig 4a

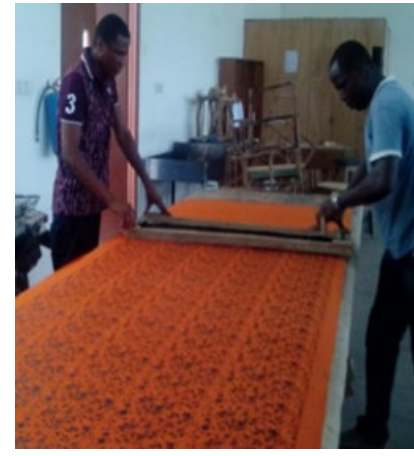

Fig $4 \mathrm{~b}$

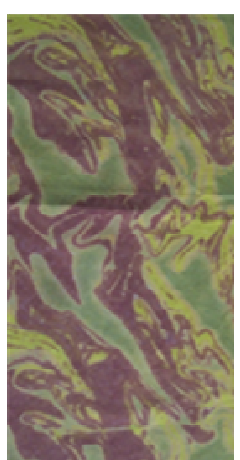

Fig 4a \& b: The printing being carried out by the researchers
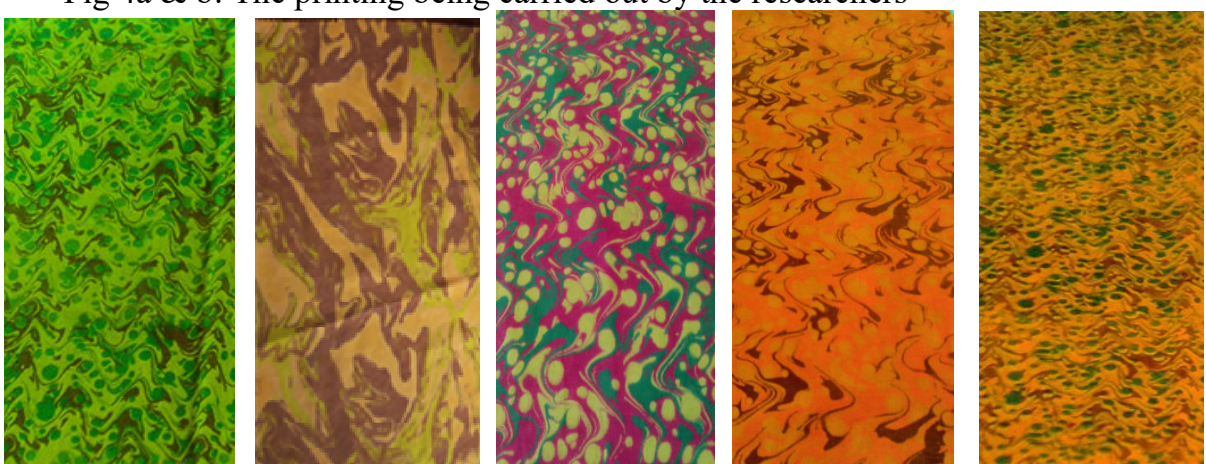

Fig. 5: These are samples of finished printed marbled designs using vat dye paste on reactive dyed background of various colours

Fashioning of Fabric Pattern into Garments and Accessories

The fabric prints have to be fashioned into garments and some accessories to assess their aesthetics and functionality values within the Ghanaian market. This part involves the designing of the garments (male and female shirts and dresses) as well as footwear and a bag to be used as accessories. The patterns for the various designs were created before the final assembling of parts into finished products were executed. The designs were done with preliminary sketches on different styles of garments and accessories. Out of which two short sleeve shirts for men and two simple dresses for female (long and short styles) were selected for the products. A simple ladies bag and footwear were also selected and fashioned into the accessories. Patterns for the various products were designed with the help of technicians in the field of leather work for the drafting and construction of the bag and the footwear. The patterns for the garments were drafted with the necessary allowances for easy fitting. The patterns were later cut together with the garment fabric. Constructions of the garments were carried out in the studios of the researchers with the help of research assistants. Colour matching, good finishing procedures and ease for comfort in wear were all considered during the production of the garments. The finished products were worn by models as exhibited in figure $6 a, b, c$ and d. Figure 7 shows the bag and footwear produced. 


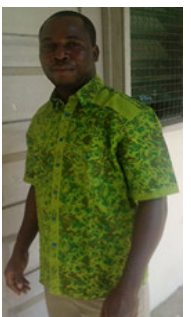

Fig. 6a

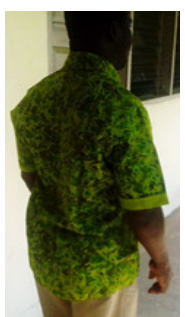

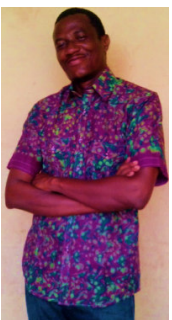

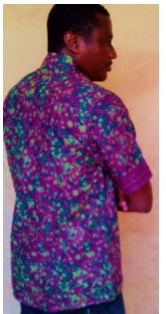

Fig. $6 b$

Figure $6 \mathrm{a} \& \mathrm{~b}$ : Male models exhibiting two different garment designs produced from the reactive dyed background and vat dyes paste screen printed fabrics.

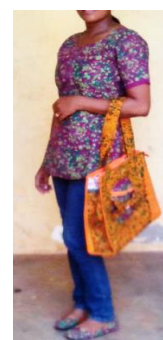

Fig. 6c

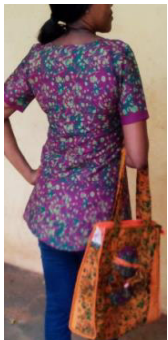

Fig.

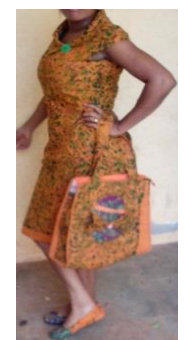

Fig. 6d

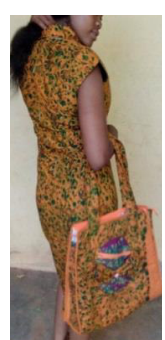

Figure $6 \mathrm{c} \& \mathrm{~d}$ : A female model exhibiting two different garment produce from the designs produced from the reactive dyed background and vat dyes fabric print samples paste screen printed fabrics.

\section{Results and Discussion Main findings}

Generally, the outcome of the experiment was successful. The series of exploration conducted with the various starches as thickening agent reveal that wheat flour starch produces a smoother paste hence the best result of prints among the rest used for the dye paste preparation. The consistency of the paste prepared is crucial in the volume of dye penetration onto the fabric which determines the shade and the fixation capabilities of the fabric when printing. The consistency should be such that it will enhance easy penetration into the fabric but should not be too watery as it will lead to excessive migration of the colours into unwanted areas and distort the beauty of the design.

The finding also revealed that various colours printed on the fabric with the vat dyed paste only appear as dark shades on the fabrics until full oxidation occurred and the fabrics washed before its true colour shades are revealed. This comes with the challenge of determining the true shades of colours produced at the time of printing. The researchers also identified that, the time between the printing and washing off of excess dyes to expose the result is crucial in determining the outcome and durability of the printed fabric. A shorter duration of time between the after-print, oxidation and washing-off of excess dyes will not produce good shades of colours as dye absorption into the amorphous regions of the fibres might not be exhausted while a longer duration will equally weaken the fibres due to the presence of the sodium hydroxide and the bleaching agent added in the dye preparation. It was however realised that an average time of 45 to 60 minutes is good enough for acceptable results.

The series of samples carried out were designed to explore difference in shades of background colours and vat dye paste produced. A typical finding was that colours are not bright as expected, especially with regards to the vat dye print paste. The advantage of this printed technique is that, it eliminates the heavy visible feels associated with screen printed fabrics carried out in small batches by small and medium scale producers in Ghana which does not make locally printed fabrics attractive. The various forms of marbling effects produced on fabrics have challenges with sharp edges of designs involved; reproduction of such designs when the need be and sometimes the resultant colour effects are not too encouraging. These are the challenges that the researchers intend addressing with larger percentage of success in exception of the non-vivid shades the vat dye paste produces. Since Ghanaians are noted for the functionality of their fabrics, the design produced into the garments was named as 'there is good life ahead of us' 'abrabopa wo yen anim'. The finished fabric was evaluated by a panel of final year students and lecturers with clothing and textiles background.

\section{Evaluation of the printed sample fabrics}

The evaluation was done in reference to fabric characteristics and performances relating to texture, hand, bulk and durability as in colourfastness. This rating was done in comparison to other forms of hand screen printed fabrics using the likert scale. Twenty panelists with textiles and fashion background were asked to rate the sample in order of Excellent, Very Good, Good, Fair and Poor. From the evaluation, all panelists rated the 
sample fabric as excellent in hand (that is the feel of the fabric between the fingers) and texture (that is the surface look of the fabrics). Bulk was assessed based on the weight of a two yard length of sample fabrics compared with hand screen printed ones using simple digital scale. This actually considered the amount of print paste deposition on the fabric which affect stiffness and drape of the fabric. In this instance, the quality of work is determined by the lightness in weight of the fabric. Seven panelists ticked Good, eleven ticked Very Good, one each ticked Fair and Excellent respectively. It implies that, this printed fabric samples are lighter in weight and will enhance drapability better than the other hand printed ones in the market. Colour fastness was considered under durability to determine how fast the dyes stayed or run on the fabric after series of washing. With this, five panelists ticked Good, nine ticked Fair and the rest of the six ticked Poor, showing their dislike with the running abilities of the fabric. However, the fourteen others partially acknowledge the colour fastness properties of the fabric but it is clear that, they believe some amount of work need to be done in improving the fastness of the dyes especially the reactive dyes for better results.

\section{Conclusion}

The printed work exhibits the intended ideas it was designed for as it truly replicate effects of marbling techniques using vat dyes as paste based medium. The research was designed to provide alternative means for screen printing pastes used locally that deposit heavy surface effects on the fabrics printed making them unattractive to buyers. The use of vat dye based paste produces effects with similarity to industrial prints in Ghana.

The results provides alternative to textile students, educators and small scale producers of fabric prints to improve on their screen printed works using vat dyes based pastes which can be easily prepared by anybody in this field. The technique will equally provide additional option for batik and tie-dye producers to generate and print these types of designs for an alternative result or combined the technique with their batik works, especially when designs with accurate edges are desired for specific end results.

\section{Recommendations}

In view of the vast potentials it offers for educators, students and small scale producers in the field of textiles, the following suggestions are proposed for consideration. Firstly, students and educationist in textiles should be encouraged to explore the possibility of using the techniques and to improve on the brightness of colours produced especially using reactive dyes as alternative to the vat dye paste.

The researchers also called for further studies on colour mixing and matching techniques for vat dyes when using them to prepare the paste, as the combination of two or more vat dye colours and their intended shades when mixed do not usually produce the required results.

\section{References}

Adu-Akwaboa, S. (1989). Art for Schools and Colleges. Samarg Publications: Kumasi. p. 41.

Asante, C.K., Nyarko-Hibson, J. A. (2008). Graphic Design for West African Senior High Schools and Colleges.

Rock of Salvation Publishers: Kumasi. pp. 1, 13, 17, 112.

Asare, K. D. (2010). The Use of Asante Linguist Staff Symbols in Textile Design, KNUST, Kumasi: $M F A$ Thesis, Department of Industrial Art. pp 6-10

Batik-Octoro., (2019). Marbling. Website; www.dyerman.com/products/marbling. Retrieved on; 05/11/2019

Bette, J. D. (1997). Marbling Magic, University of Kentucky, Lexington-USA: College of Agriculture. p. 1.

Brett, P. (1990). Carpentry and Joinery for Building Craft Studies I. Cheltenham: Stanley Thornes Publisher Ltd.

Best, J. W. (1981). Research in Education. Englewood, USA: Prentice Hall Inc.

Chambers, A. (1995). Marbling on Fabric, Search Press: United Kingdom.

Dharma trading co. (2019). Marbling basics: A step-by-step guide to marbling fabric. Website;www.dharmatrading.com/techniques/marbling-instruction.html. Retrieved on 05/10/2019.

Dzramedo, B. E., Ahiabor, R. \& Gbadegbe R. (2013). The Relevance and Symbolism of Clothes within Traditional Institutions and Its Modern Impacts on the Ghanaian Culture. International Institute for Science, Technology and Education; Arts and Design Studies (ISSN 2224-6061 (print), vol. 13 pp 1-13.

Fatma, G. (2015). TV Advertisement and Eating Habits of Children - The Two Inseparable Strings. International Journal of Innovative Research and Advanced Studies (IJIRAS), Vol. 2, Issue 5, pp 18-20.

Galen, B. (2015). The Ancient Art of Marbling on Paper and Fabric. Retrieved from marbleart.us. on $28^{\text {th }}$ May, 2015.

Howard E. K. (2013). Exploration of Mixed-Media Techniques in Textile Art. The International Journal of Humanities and Social Studies (ISSN 2321-9203): www.theijhss.com. p. 12.

Humphries, M. (2004), Fabric Reference. ( $3^{\text {rd }}$ Ed.), New Jersey, Pearson Education Inc.

Kadolph, S. J. (2007). Textiles. (10th Ed.), New Jersey, Upper Saddle River: Pearson Prentice Hall,. pp 148-149

Manu A. (2010). Hairstyles for Fabric Designing and Production, KNUST, Kumasi: MFA Thesis, Department of 
Industrial Art. p. 6.

Pazar, C. E. (2016). The progress of the Art: Paper Marbling in the United States, 1880 - 1950. Ann Arbor; Pro Qest LLC. Pp 12 - 18.

Shaeffer, C. (2001). Sewing for the Apparel Industry. New Jersey: Upper Saddles River-Prentice Hall, Inc. Page $1-18$.

Singh, S. (2006). Impact of Color on Marketing. Current Research Development. www.emeraldinsight.com/0025-1747.htm. Pp 783 -789.

Tortora, P. G. \& Merkel, R. S. (2007), Fairchild Dictionary of Textiles. (7th Ed.), U.S.A.: Fairchild Publications.

Weston. T. P. (2006) http://www.fashion-era.com Retrieved on 22nd of December, 2010

Wilson J. (2001). Handbook of Textile Design: Principles, Processes and Practice. England: Woodhead Publishing Ltd. p. 17.

Wolfe, G. M. (1989). Fashion! A Study of Clothing Design and Selection; Textiles, the Apparel Industries and Careers. South Holland. Illinois: Goodheart Willcox Company, Inc.

First Author: Bernard Edem Dzramedo obtained a BA (Hons) in Industrial Art with Textile option from Kwame Nkrumah University of Science and Technology (KNUST), Kumasi; an Mtech in Textile and Fashion Design from University of Education (UEW), Winneba, Kumasi Campus and an Mphil/PhD degree in African Art and Culture from Kwame Nkrumah University of Science and Technology (KNUST), Kumasi. He is a Senior Lecturer and currently the Head of Department for Family and Consumer Sciences at the University for Development Studies, Nyankpala campus - Tamale in the Northern Region of Ghana.

Second Author: Robert Ahiabor obtained his first degree in BA Industrial Art (2007) and MFA in Textile Design in 2011 all from University of Science and Technology (KNUST), Kumasi in Ghana. Robert is currently a Senior Lecturer at the department of Fashion Design and Modelling and the Dean of School of Applied Art, Design and General Studies at Wa Polytechnic. 